\title{
Flexible Support Measures and Search Schemes for Mining Frequent Patterns in a Large Single Graph
}

\author{
Jinghan Meng \\ Department of Computer Science \& Engineering \\ University of South Florida \\ jmeng@mail.usf.edu
}

\begin{abstract}
Recent years have witnessed intensive studies on mining graph databases for interesting patterns. One important problem in this paradigm is the Frequent Subgraph Mining (FSM), which involves finding patterns that occur in high frequency from a graph database. In particular, researchers are interested in finding all frequent patterns from a single graph that often consists of a large number of vertices and edges. There are two indispensable components in any solutions to the FSM problem. The first one is a support measure that quantifies the frequency of a given pattern occurred in the data graph. The second one is an algorithm for searching through the space of all possible subgraphs to identify frequent ones. These two computations, if handled in a naive way, face serious performance and accuracy issues. The main objective of my research project is to study both processes by providing theoretical breakthroughs and developing practical algorithms to further speed up the processing of FSM.
\end{abstract}

\section{Keywords}

Data mining; graph mining; hypergraph

\section{INTRODUCTION}

Graphs have become increasingly important in modeling complicated structures such as chemical compounds, biomolecules and metabolic networks, social networks, aviation maps, and the Web. As shown in many applications, frequent patterns are believed to reveal essential features of the modeled system. In this proposed project, we focus on the frequent subgraph mining (FSM) problem. Conceptually, FSM problem needs to be processed by enumerating a large number of (if not all) subgraphs and counting their support (or frequency) to see if they are above some minimum support threshold. The identified subgraphs are called frequent patterns of the input data graph. FSM problems can be divided into two categories based on the database

Permission to make digital or hard copies of part or all of this work for personal or classroom use is granted without fee provided that copies are not made or distributed for profit or commercial advantage and that copies bear this notice and the full citation on the first page. Copyrights for third-party components of this work must be honored. For all other uses, contact the owner/author(s).

SIGMOD'17 Student Research Competition May 14-19 2017, Chicago, IL, USA

(C) 2017 Copyright held by the owner/author(s).

ACM ISBN 978-1-4503-4199-8/17/05.

DOI: http://dx.doi.org/10.1145/3055167.3055177 settings. In the graph-transaction FSM setup, frequent patterns are to be found from a database comprising of multiple (small) graphs. In the past decade, fruitful research has been published in the transaction-graph database setting: a few representative publications include Borgelt and Berthold [3], Yan and Han [13], Inokuchi et al. [7], Huan et al. [6], Kuramochi and Karypis [9]. The FSM problem is more interesting and challenging in a single-graph setup, in which the database is only one graph that often consists of a large number of vertices and edges. However, FSM in a single large graph setting has not been fully addressed, only a few reports (e.g., Kuramochi and Karypis [8, 9], Elseidy et al. [5]) can be found. There are also studies addressing similar problems but formulated in a different way: [14] studied top-K large frequent pattern mining handling various constraints in addition to frequency requirement, [1] developed methods for representative pattern mining (in transactiongraph setting). My research explores solutions for the traditional FSM problem, i.e., finding all frequent patterns within a large single data graph.

\section{APPROACH AND CONTRIBUTIONS}

My research project is presented in the following two thrusts. (1) Defining and Analyzing Support Measures in a Unified Framework: A clear definition of any FSM problem depends on a support measure as a notion of the frequency of the patterns of interest. Counting the frequency of a pattern in a graph is a very technically challenging problem due to various semantical requirements and consideration of computational overhead. In [10] my advisor and I proposed an approach for defining and analyzing a multitude of support measures based on the concept of occurrence / instance hypergraph, which unifies existing minimum-image (MNI) [4] and overlap-graph support measures (MIS)[11, 12]. Hypergraph [2] is one of the most important discrete mathematics subjects that has significant applications in data mining. Basically, a hypergraph $H=(V, E)$ consists of a set $V=\left\{v_{1}, v_{2}, \cdots, v_{n}\right\}$ of $n$ vertices and a set $E=\left\{e_{1}, e_{2}, \cdots, e_{d}\right\}$ of $d$ edges, where each edge is a subset of $V$. In an occurrence hypergraph, $V$ is the collection of all pattern node images, and each edge $e_{i}$ is a collection of pattern node images mapped by occurrence $f_{i}$. In an instance hypergraph of pattern $P$, an edge corresponds to the set of vertices of an instance of $P$. An example of occurrence and instance hypergraph can be seen in Figure 1.

Based on the hypergraph concept, we have defined two new support measures: the minimum instance (MI) measure and the minimum vertex cover (MVC) measure. For 
the MI support measure, we show that the existing MNI support is an upper bound for it, or in other words, it is closer to the MIS support of a pattern than the MNI. Same as MNI, the MI support is also linear-time computable. The MVC support returns frequency that is even closer to MIS. Although computing MVC measure is NP-hard, it enjoys a $k$-competitive approximate algorithm where $k$ is the number of nodes in the pattern.

Under the hypergraph framework, we provide polynomialtime computable relaxations of both MVC and MIS measures. This makes MVC and MIS more efficient while still providing meaningful frequency values.

In summary, by the value (denoted as $\sigma$ ) returned for the same data graph and pattern, the aforementioned support measures can be ordered as

$$
\sigma_{M I S} \leq \sigma_{M V C} \leq \sigma_{M I} \leq \sigma_{M N I}
$$

As to computational complexity, MIS and MVC are both NP-hard while MI and MNI are both linearly computable. Therefore, the gap between the NP-hard and linear-time measures is still large. Luckily, the hypergraphs, by capturing a rich set of topological information of the pattern's appearance in the graph, allow us to define new flexible support measures in an unified framework. Our preliminary work has demonstrated the power of this framework by introducing several novel support measures with desirable features. I plan to present analysis of approximation algorithms for existing NP-hard measures by utilizing fruitful results in hypergraph theory. Another task is to define the first non-relaxation polynomial time support measures filling the gap between NP-hard MIS/MVC and linear-time $\mathrm{MI} / \mathrm{MNI}$ in terms of computation complexity and support count. Furthermore, I will give sufficient conditions for producing user-defined linear-time measures, and study variations of existing support measures with other useful features (e.g., relaxed overlap concept, parallel computing) within this framework.

(2) Novel Pattern Search Algorithms: Most of the existing single-graph FSM algorithms explore a large number of candidate patterns. The pattern exploration usually starts with smallest patterns and then extend to bigger ones. Considering the huge search space, such schemes that visit patterns in a top-down manner are not feasible when dealing with large data graphs. I am developing a new pattern generation algorithm and search scheme for FSM in large graphs. By exploring the candidate patterns in a "bottomup" style, my algorithm effectively trims the search space to achieve much higher efficiency. I will utilize the newly developed support measures and use sophisticated statistical and hypergraph methods to guide the traverse of the candidate tree and analyze the performance and accuracy guarantees of our algorithm. I also plan to develop incremental algorithms for FSM problems defined over graph data streams and under different frequency thresholds.

The entire algorithm can be divided into three stages:

I: Top-down DFS Pass: We traverse the pattern search tree in a DFS manner until it has exhausted growth options of the edge with the lowest lexicographic order. In Fig 2, the pattern we find in the first pass are shown in the pink area.

II: Find Maximal Patterns on the Perimeter of Pattern Trees: A frequent graph $G$ is maximal if there exists no supergraph of $G$ that is also frequent. From the

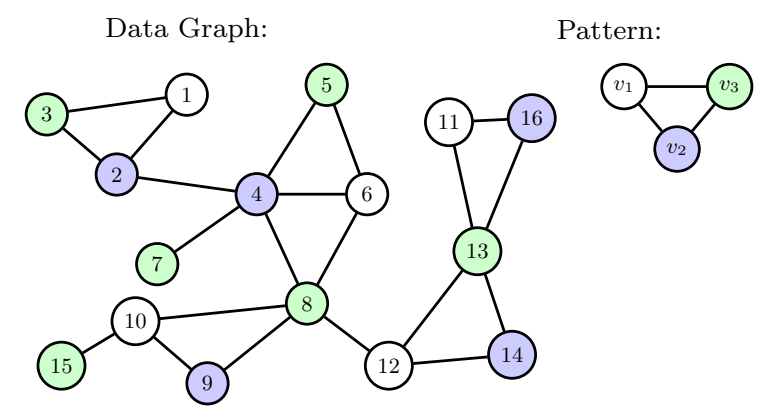

Occurrence/Instance Hypergraph:

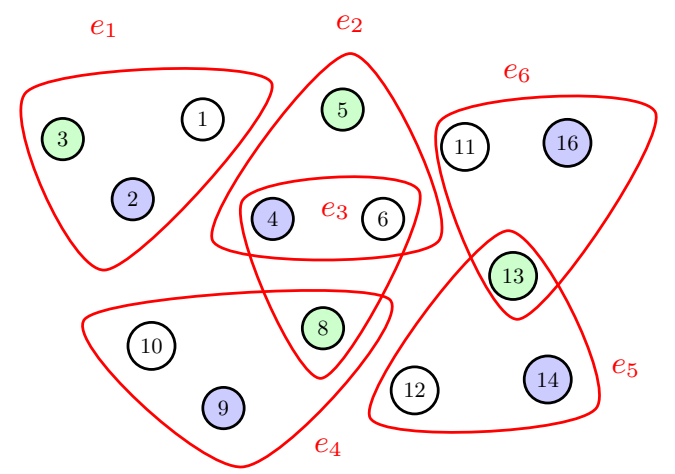

Figure 1: Occurrence/instance hypergraph of a triangular pattern

set of maximal frequent pattern discovered in Stage I, we try to find more neighboring maximal frequent pattern hence avoid searching the inner part of pattern trees.

III: Maximal Patterns Recovery: A number of large maximal frequent patterns have been discovered, hence we discovered a rough version of the frequent perimeter of the pattern trees. We only need to go over them to check if there are missing maximal patterns. Most likely they are smaller maximal patterns because we have roughly searched on the perimeter of these pattern trees. In Figure 2, the green patterns $a, b, d, e, n, o$ are maximal patterns.

Algorithm Analysis: The proposed optimizations help reduce candidate generation and unnecessary support computation, drastically reduce the search space from the whole pattern tree to its perimeter. I will develop an analytical model to quantify the performance of our algorithm.

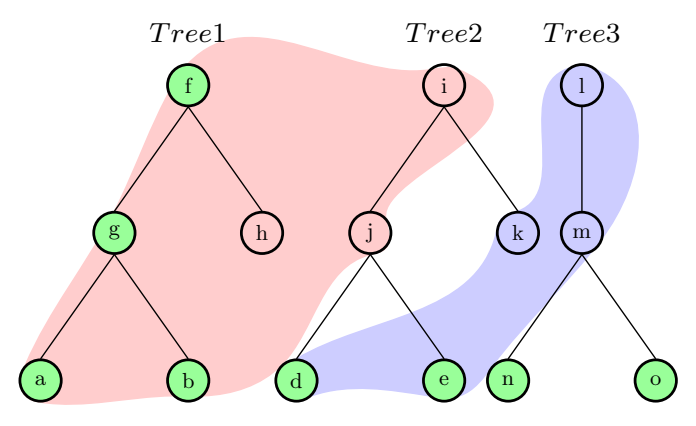

Figure 2: Perimeter Search Scheme 


\section{REFERENCES}

[1] M. Al Hasan, V. Chaoji, S. Salem, J. Besson, and M. J. Zaki. Origami: Mining representative orthogonal graph patterns. In Seventh IEEE international conference on data mining (ICDM 2007), pages 153-162. IEEE, 2007.

[2] C. Berge. Hypergraphs: combinatorics of finite sets, volume 45. Elsevier, 1984.

[3] C. Borgelt and M. R. Berthold. Mining molecular fragments: Finding relevant substructures of molecules. In Data Mining, 2002. ICDM 2003. Proceedings. 2002 IEEE International Conference on, pages 51-58. IEEE, 2002.

[4] B. Bringmann and S. Nijssen. What is frequent in a single graph? In Pacific-Asia Conference on Knowledge Discovery and Data Mining, pages 858-863. Springer, 2008.

[5] M. Elseidy, E. Abdelhamid, S. Skiadopoulos, and P. Kalnis. Grami: Frequent subgraph and pattern mining in a single large graph. Proceedings of the VLDB Endowment, 7(7):517-528, 2014.

[6] J. Huan, W. Wang, and J. Prins. Efficient mining of frequent subgraphs in the presence of isomorphism. In Data Mining, 2003. ICDM 2003. Third IEEE International Conference on, pages 549-552. IEEE, 2003.

[7] A. Inokuchi, T. Washio, and H. Motoda. Complete mining of frequent patterns from graphs: Mining graph data. Machine Learning, 50(3):321-354, 2003.
[8] M. Kuramochi and G. Karypis. Grew-a scalable frequent subgraph discovery algorithm. In Data Mining, 2004. ICDM'04. Fourth IEEE International Conference on, pages 439-442. IEEE, 2004.

[9] M. Kuramochi and G. Karypis. Finding frequent patterns in a large sparse graph. Data mining and knowledge discovery, 11(3):243-271, 2005.

[10] J. Meng and Y. Tu. Flexible and feasible support measures for mining frequent patterns in large labeled graphs. In Proceedings of the 2017 ACM SIGMOD international conference on Management of data. ACM, 2017.

[11] N. Vanetik, E. Gudes, and S. E. Shimony. Computing frequent graph patterns from semistructured data. In Data Mining, 2002. ICDM 2003. Proceedings. 2002 IEEE International Conference on, pages 458-465. IEEE, 2002.

[12] N. Vanetik, S. E. Shimony, and E. Gudes. Support measures for graph data. Data Mining and Knowledge Discovery, 13(2):243-260, 2006.

[13] X. Yan and J. Han. gSpan: Graph-Based Substructure Pattern Mining. In Proceedings of the 2002 IEEE International Conference on Data Mining (ICDM 2002), 9-12 December 2002, Maebashi City, Japan, pages 721-724, 2002.

[14] F. Zhu, Q. Qu, D. Lo, X. Yan, and J. Han. Mining top-k large structural patterns in a massive network. Proceedings of the VLDB Endowment, 4(11):807-818, 2011. 